\title{
Detecting single electrons in IOTA
}

\author{
Giulio Stancari for the FAST/IOTA group \\ Fermi National Accelerator Laboratory \\ Workshop on Single-Electron Experiments in IOTA \\ Fermilab, November 9, 2018
}

This manuscript has been authored by Fermi Research Alliance, LLC under Contract No. DE-AC02-07CH11359 with the U.S. Department of Energy, Office of Science, Office of High Energy Physics. 


\section{Contributors}

J. Jarvis, N. Kuklev, I. Lobach, A. Romanov, J. Ruan,

J. Santucci, A. Valishev

The SRF Group in the Mechanical Support Department

The FAST Facility Department

The Accelerator Research Department 


\section{Motivation}

Detect synchrotron-light signal and characterize backgrounds in IOTA for

1. Beam diagnostics: turn-by-turn intensity monitor with wide dynamic range, from nominal intensities ( $\sim 10^{9}$ particles) down to single electrons

\section{Scientific experiments in IOTA}

- what is the time structure of radiation emission from a single electron in a storage ring? Is it random, regular, chaotic?

- is there correlation between the emission from different dipoles?

- many other ideas... (this workshop)

Stancari et al., FERMILAB-FN-1043-AD-APC 


\section{Experimental layout}

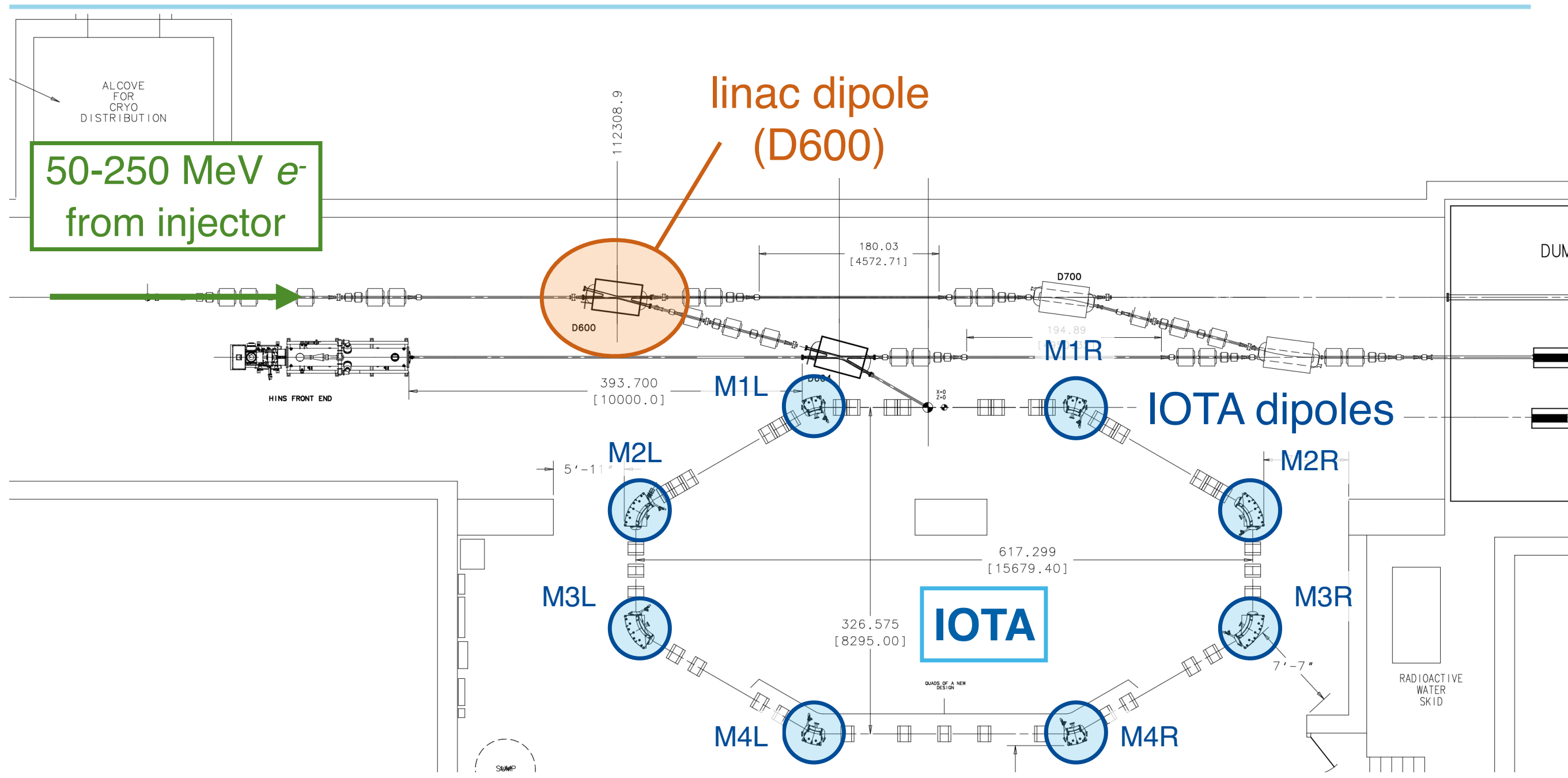

- Main dipoles instrumented with vacuum windows, light-transport periscopes, and light-tight boxes

- Synchrotron light is detected with photomultipliers and on cameras 


\section{Experimental layout in IOTA}

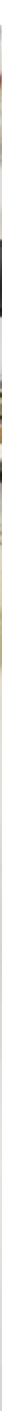




\section{Experimental layout in IOTA}
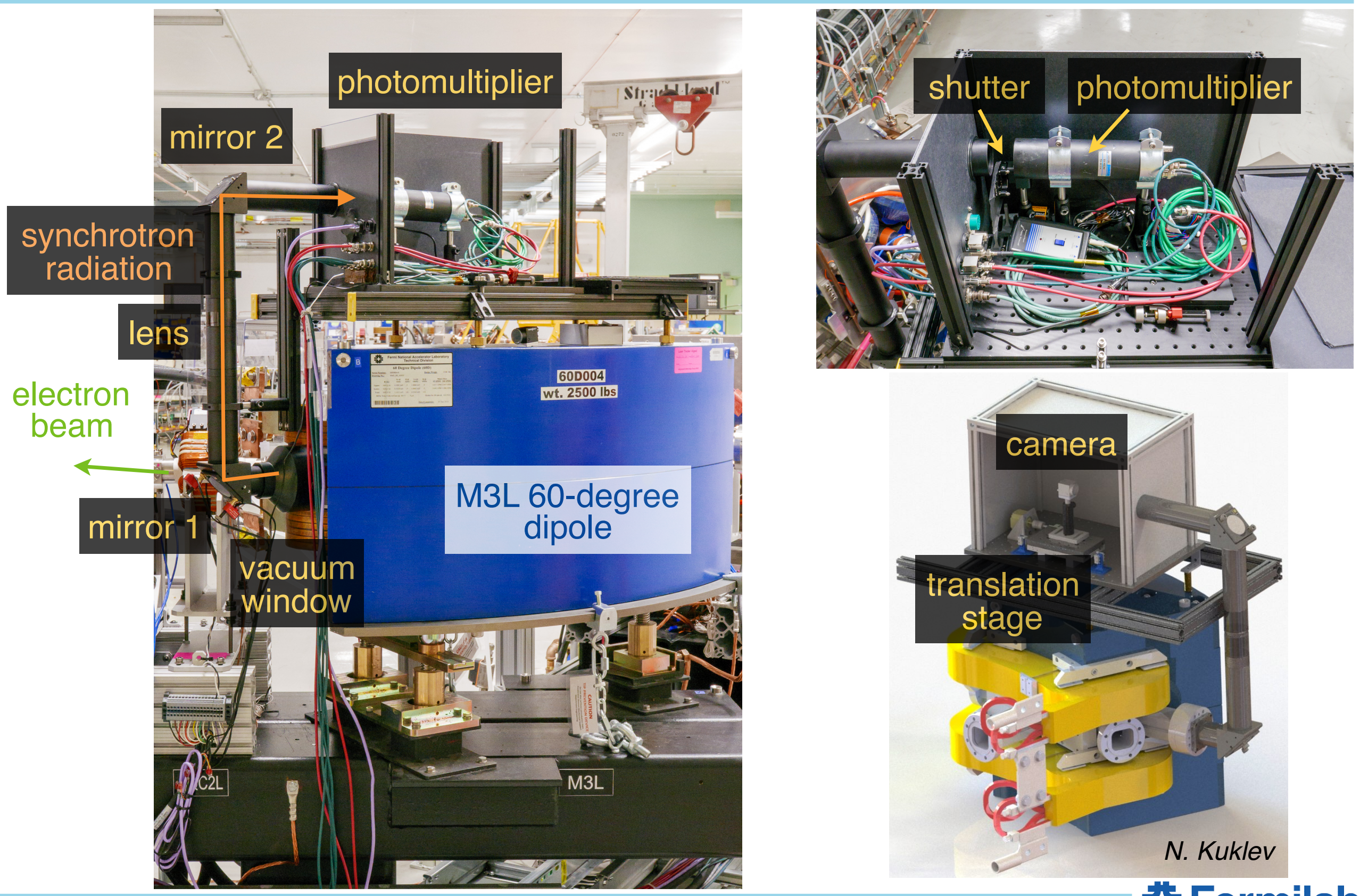

詼 Fermilab 


\section{Choice of photodetectors}

Currently, we have available

- cameras

- conventional photomultipliers (PMT):

- current mode or pulse mode to cover the full range of IOTA beam intensities

- microchannel-plate photomultipliers (MCP-PMT):

$-<100$ ps transit-time spread for timing measurements

- can be gated

- multi-pixel photon counters (MPPC, SiPM):

- pulse height allows to resolve individual photoelectrons

- very compact

- higher dark counts, sensitive to radiation 


\section{Signal processing and data acquisition schematic}

SYNCHROTRON

LIGHT

$\longrightarrow$

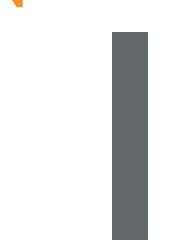

SHUTTER

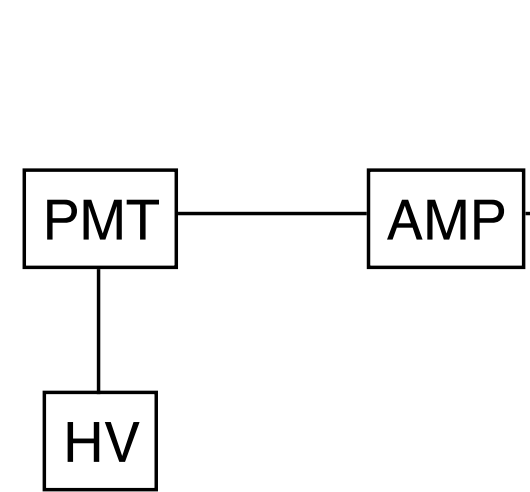

PICOAMMETER

BEAM SYNC
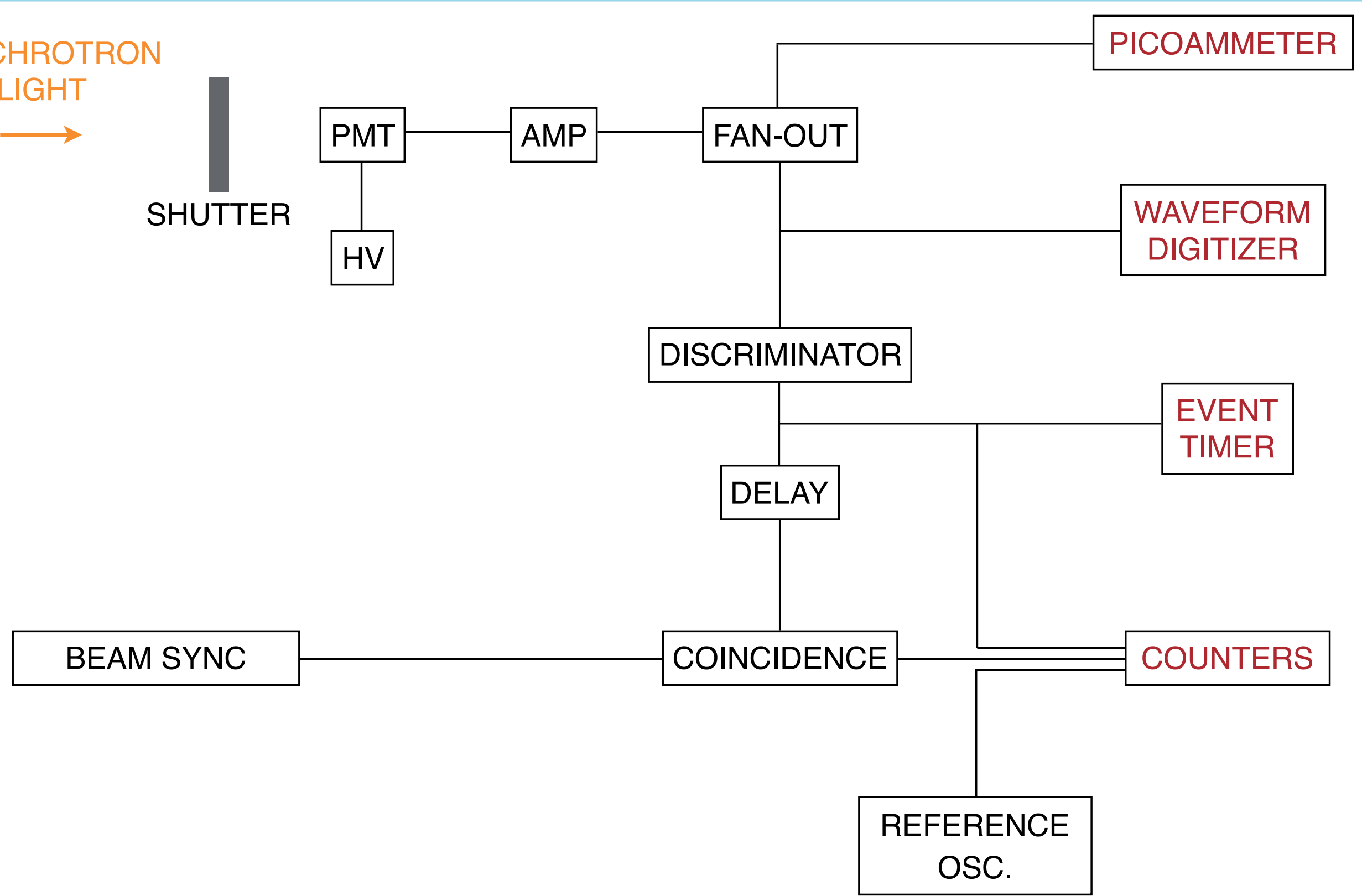


\section{Expected signal}

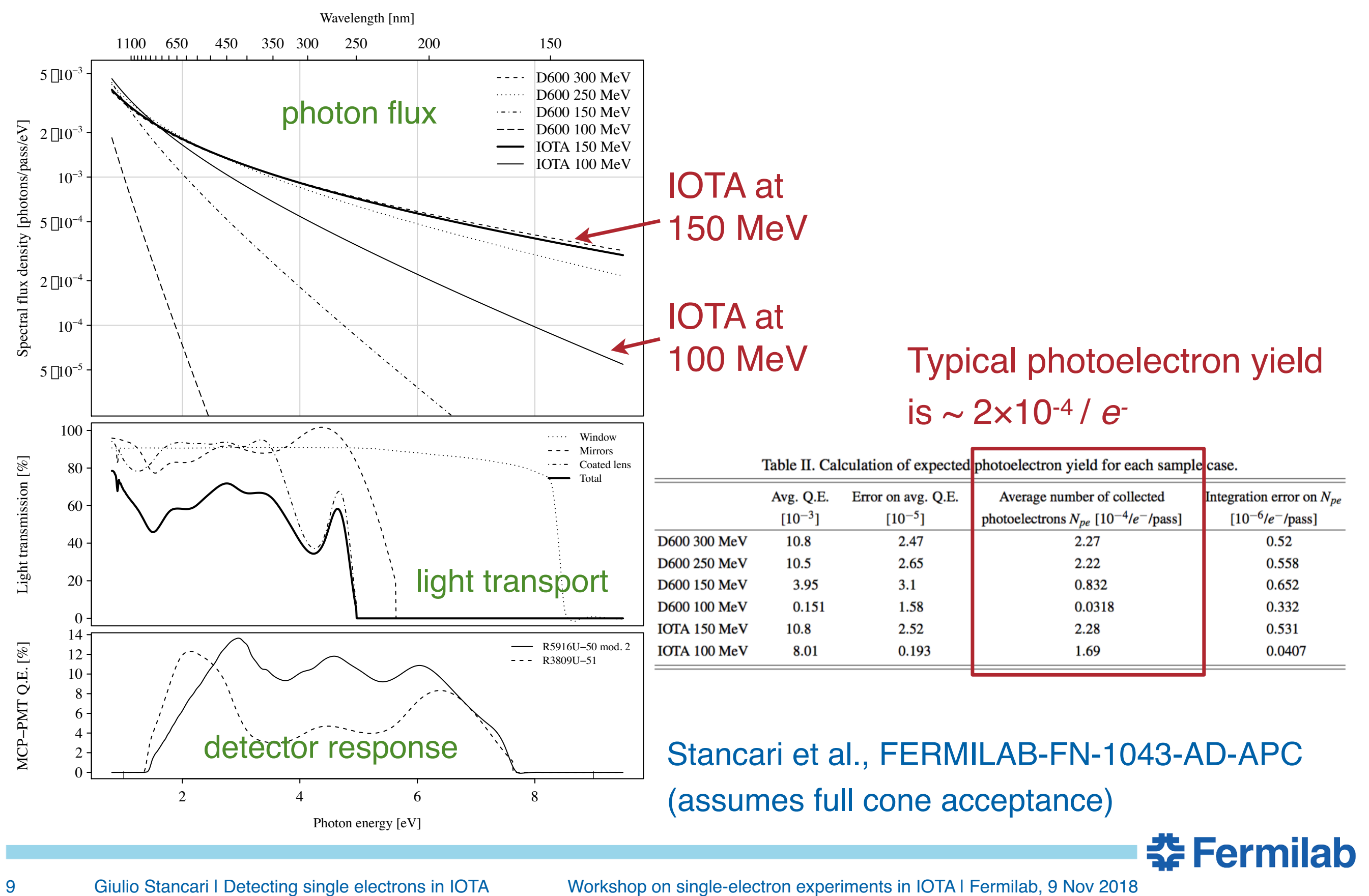




\section{Expected angular distribution of synchrotron radiation in IOTA}

Energy density per electron per pass in $(2.17,2.75) \mathrm{eV}$ band

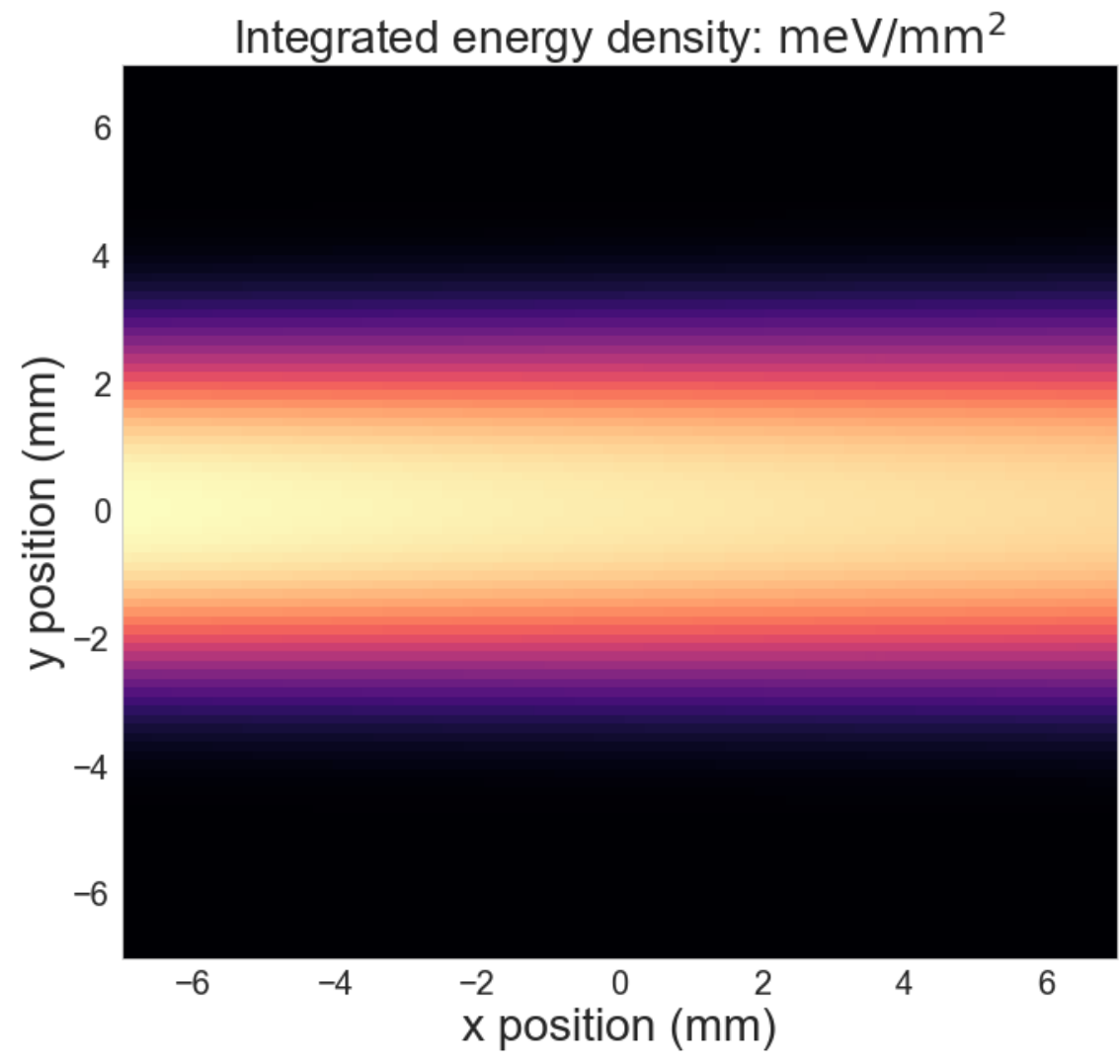

60-degree dipoles are preferred

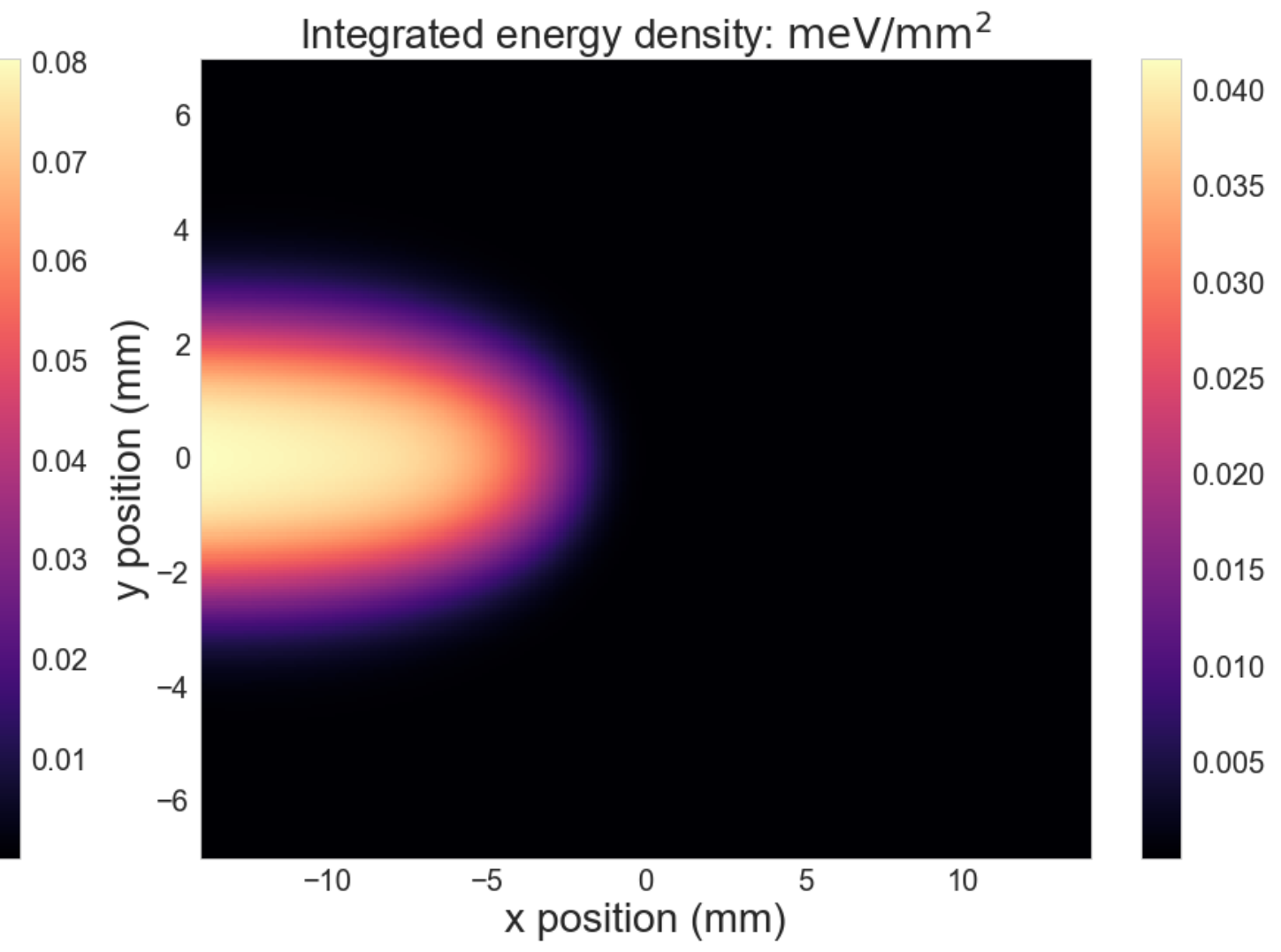

30-degree dipoles show some edge radiation

SRW calculation by J. Jarvis 


\section{Some IOTA experiments with synchrotron radiation}

Measurements carried out during the past few weeks of commissioning:

Interval between pulses, revolution period

Intensity vs. time, beam lifetime

How to achieve a known low number of stored electrons

- Wait. Test machine stability over many hours, with natural beam decay

- RF scraping: induce losses by lowering and restoring cavity voltage

- Dark linac current and detuned injection (see Romanov's talk) 


\section{Beam-based measurement of revolution period (IOTA @ $100 \mathrm{MeV}$ )}

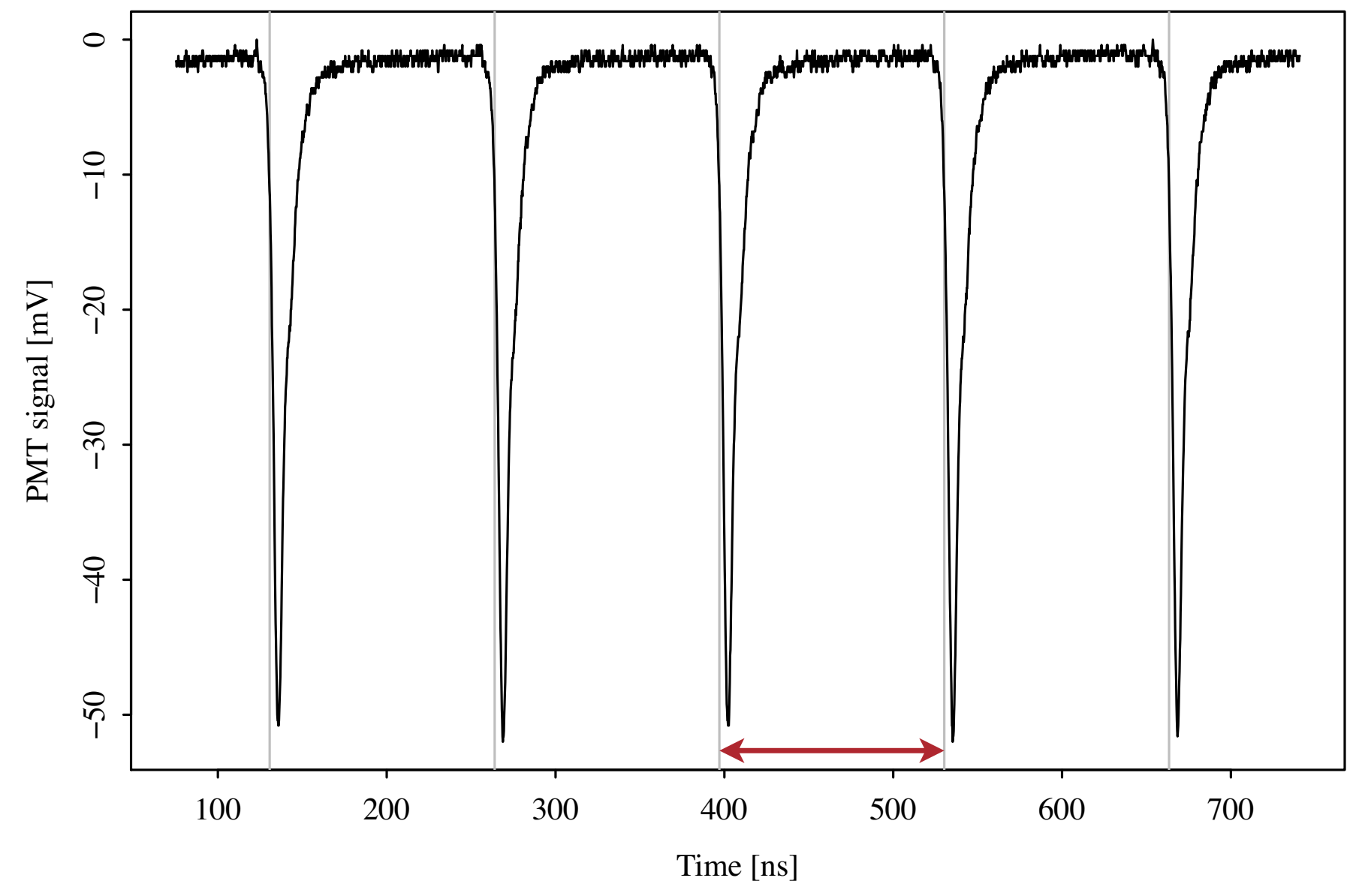

Over 598 turns, with PMT, avg. rev. period $=133172.6 \pm 2.8$ (stat.) ps (which agrees with rf cavity oscillator)

We plan to measure turn-by-turn revolution times and synchrotron oscillations, down to single electrons 


\section{Beam lifetimes over the course of a long store}

Electrons stored in IOTA from 14:00 till 22:41 on Oct. 31 PMT sensitivity study started at 17:00

Used if voltage to partially scrape the beam, then observed natural decay
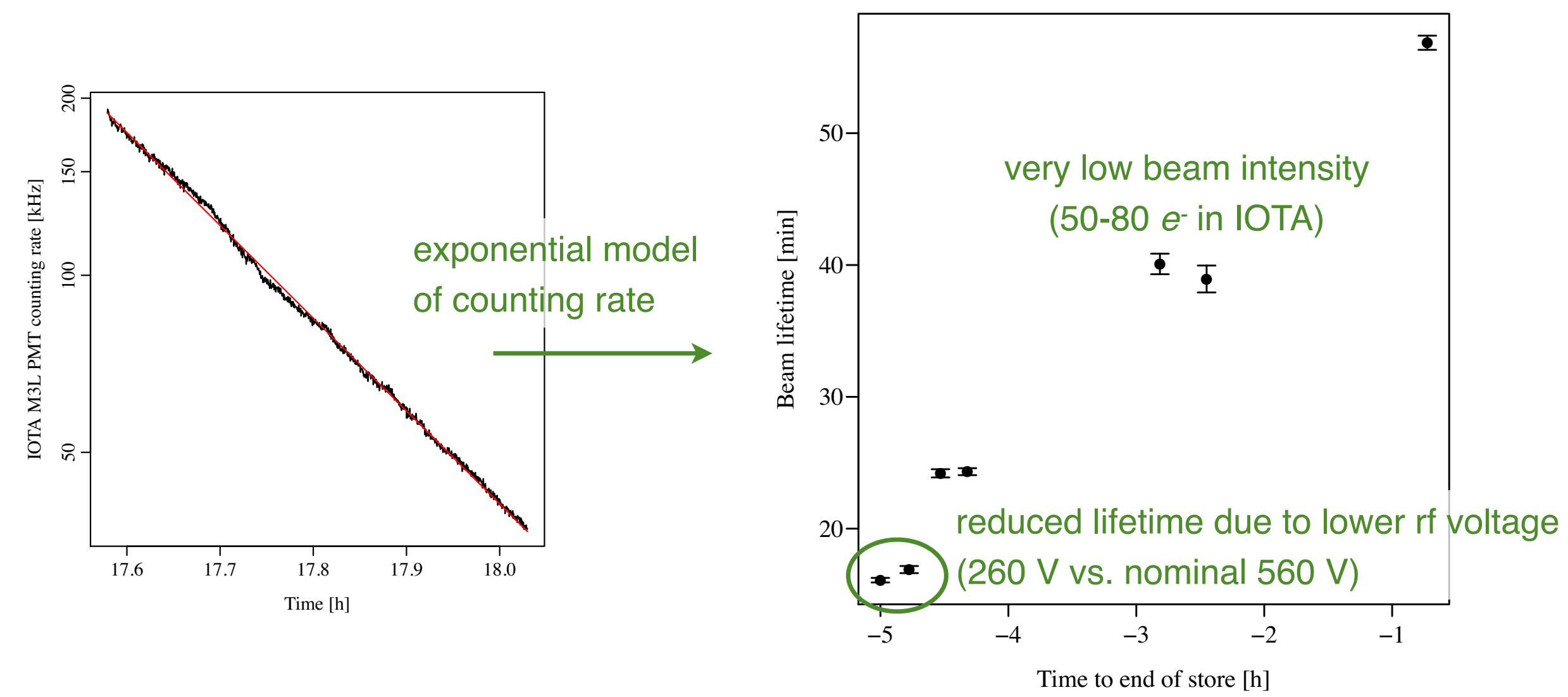

Lifetime improves with decreasing beam intensity 


\section{Decay of photon counting rate}

Towards the end of the store, one observes discrete steps in counting rate...
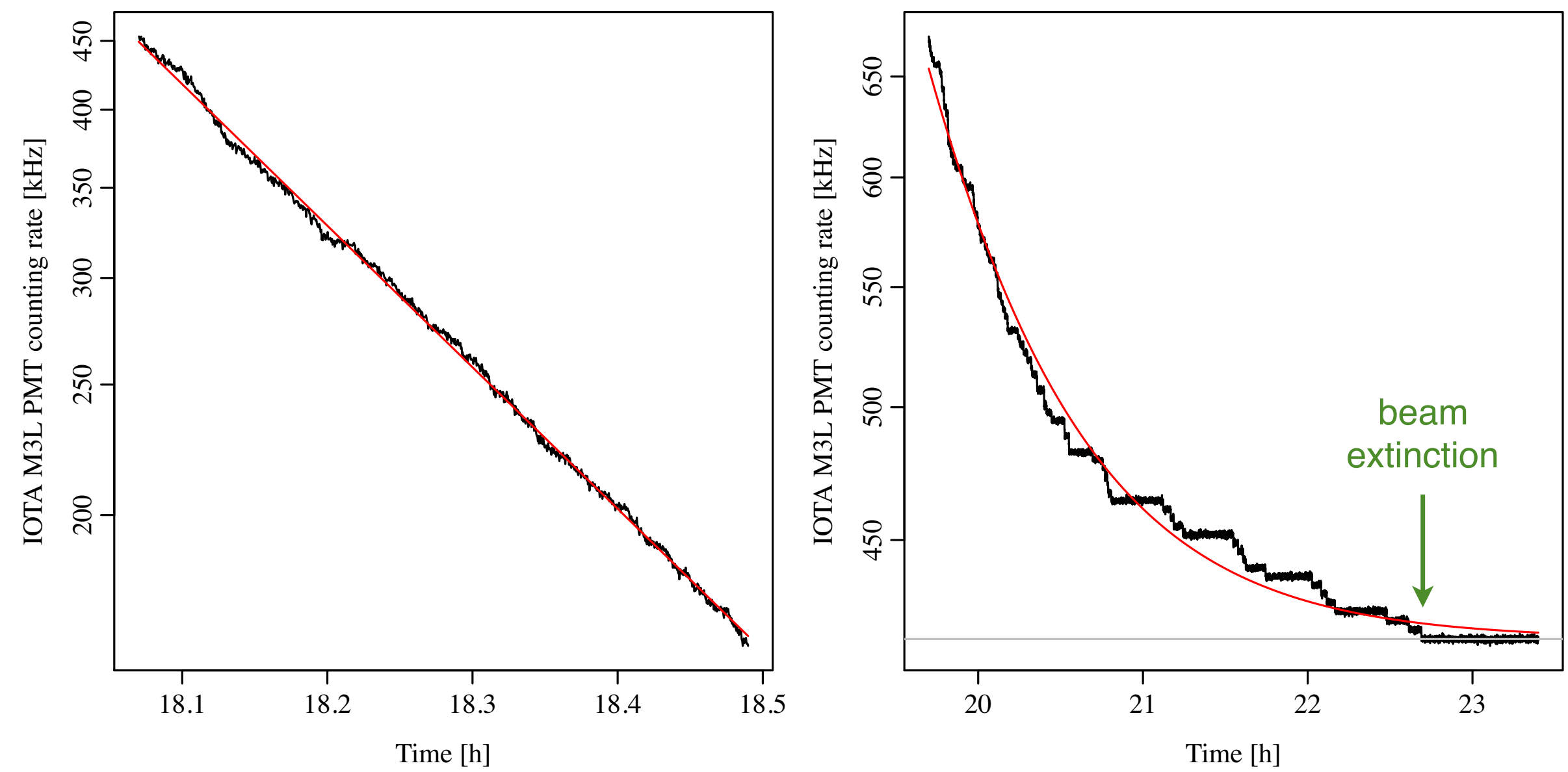


\section{Observation of discrete steps in pulse counting rates}

IOTA beam experiment of Oct. 31, 2018. Last 12 circulating electrons.

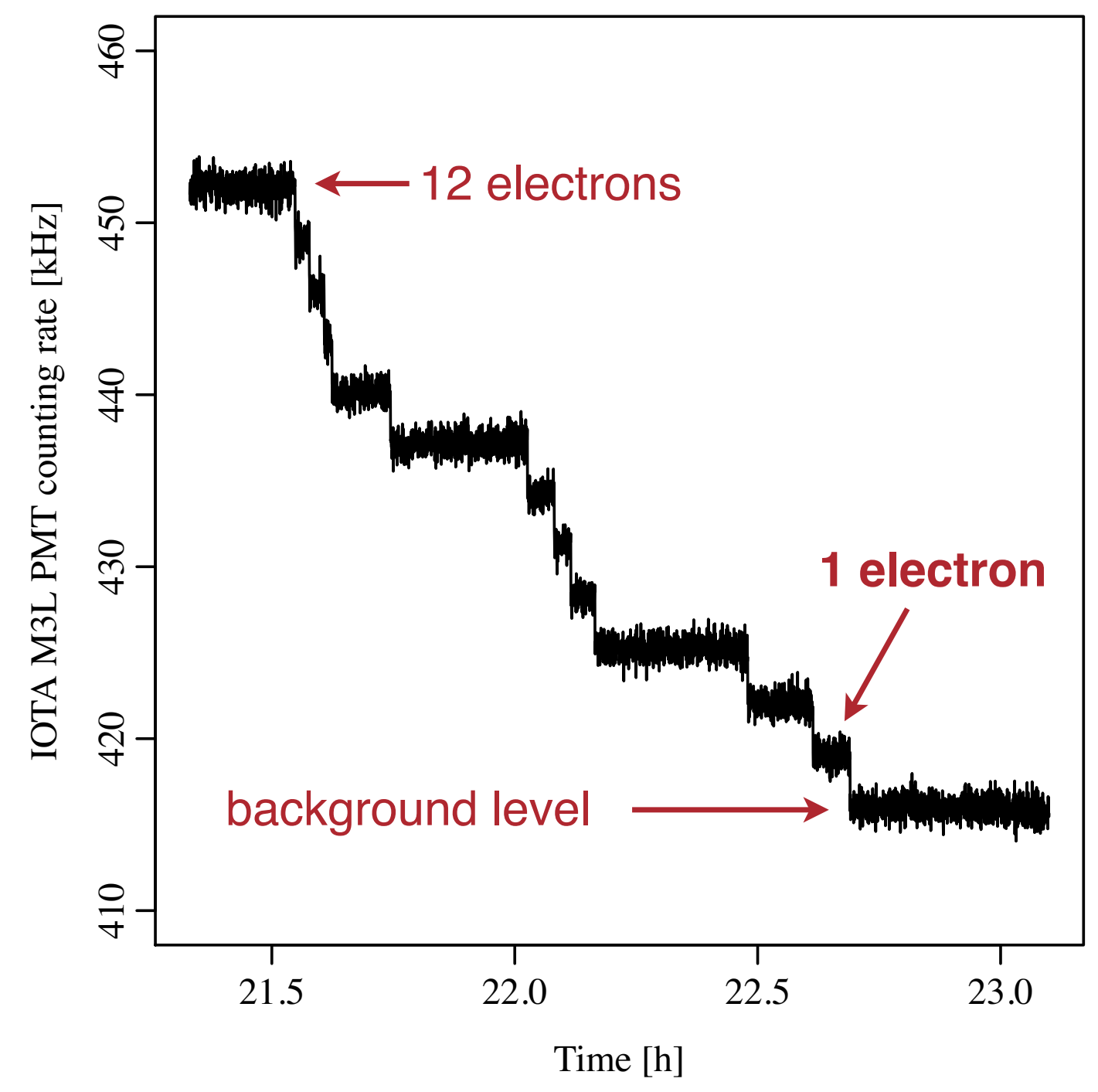

Discrete steps are multiples of $3.0 \mathrm{kHz}$, which corresponds to a single electron

Last electron circulated for 4 minutes (2 billion turns)

Single-electron experiments are possible in IOTA

We have an absolute calibration of low beam currents:

$(1.203083 \mathrm{pA}) \times N_{e}$

First observation of steps at $100 \mathrm{MeV}$ and without undulator? 


\section{Light from single electrons on camera}

Integrating over $1 \mathrm{~s}$, the cameras can see individual electrons, too!
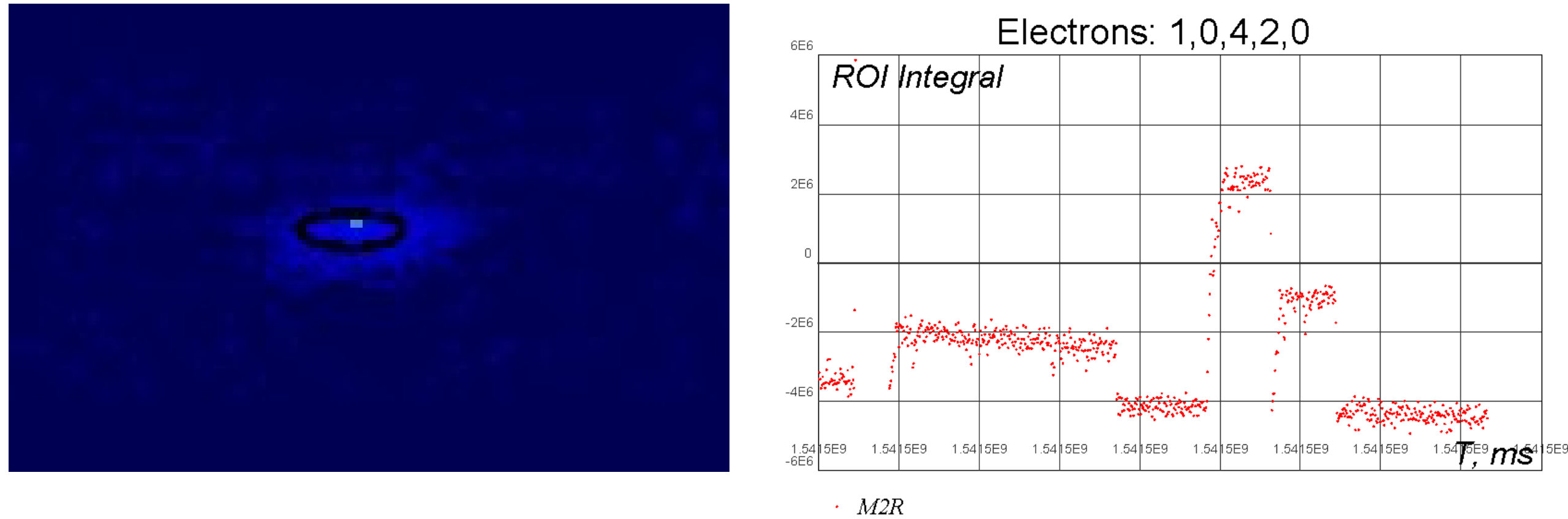

Camera intensity identifies number of stored electrons from intentionally detuned injection of dark linac current

A. Romanov 


\section{Conclusions}

After only 2 months of commissioning, we have sensitive diagnostics in IOTA to detect single electrons with both cameras and photomultipliers

A few electrons can be stored in IOTA by rf scraping (slow and coarse) and by detuned dark-current injection (faster and more reliable)

Experiments with single electrons in IOTA are definitely feasible and some have already started

Ideas and collaborations are welcome!

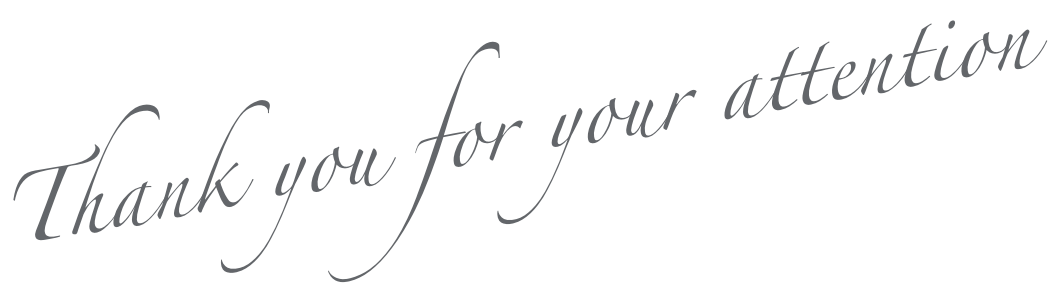




\section{Backup slides}




\section{Experimental apparatus at D600}

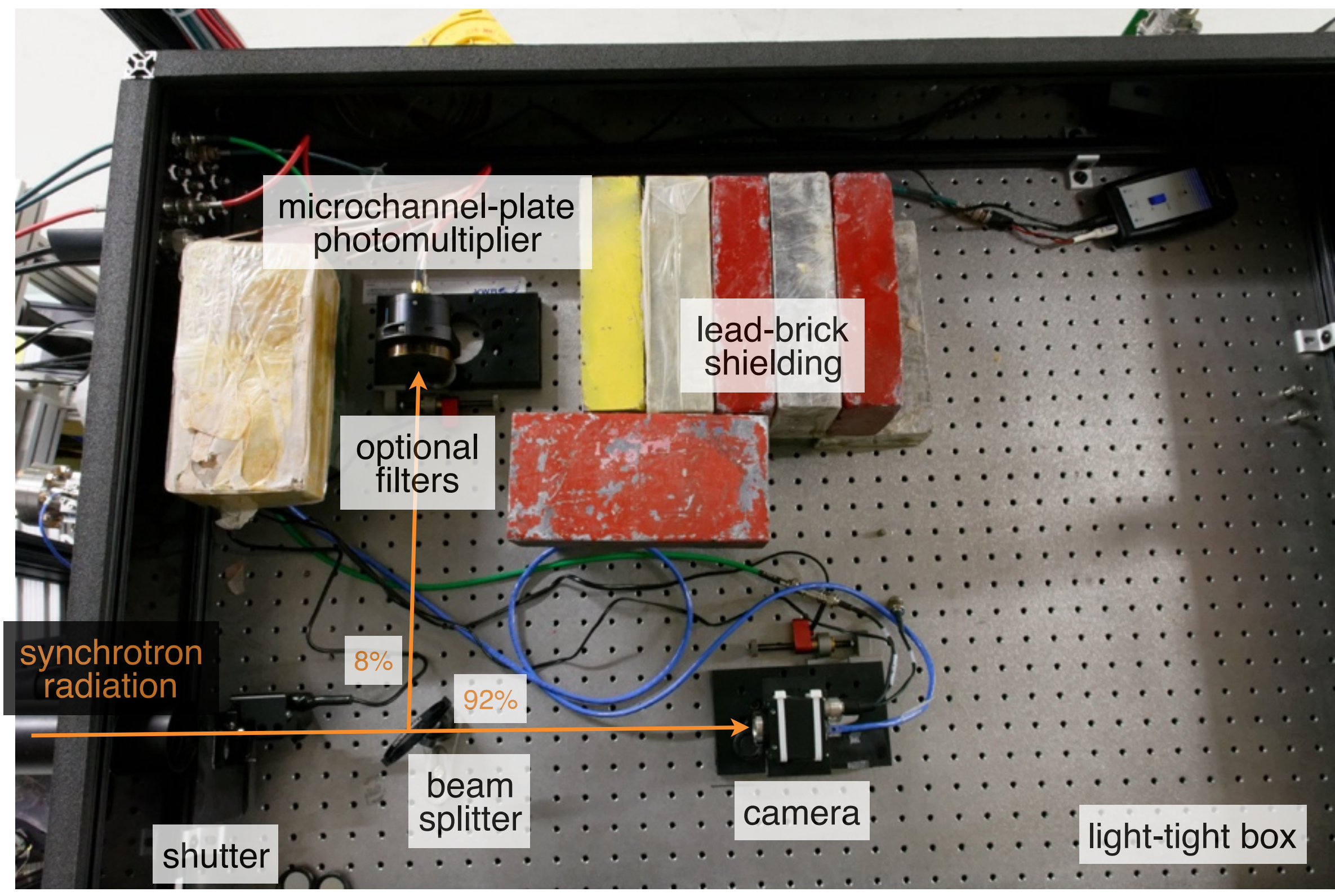




\section{Microchannel-plate photomultiplier (MCP-PMT) features}

Excellent timing (sub-ns) and high gain (103-107). Can be gated.

Limited current at high rate.

Hamamatsu R5916U-50 mod. 2 reused from Tevatron Synclite.
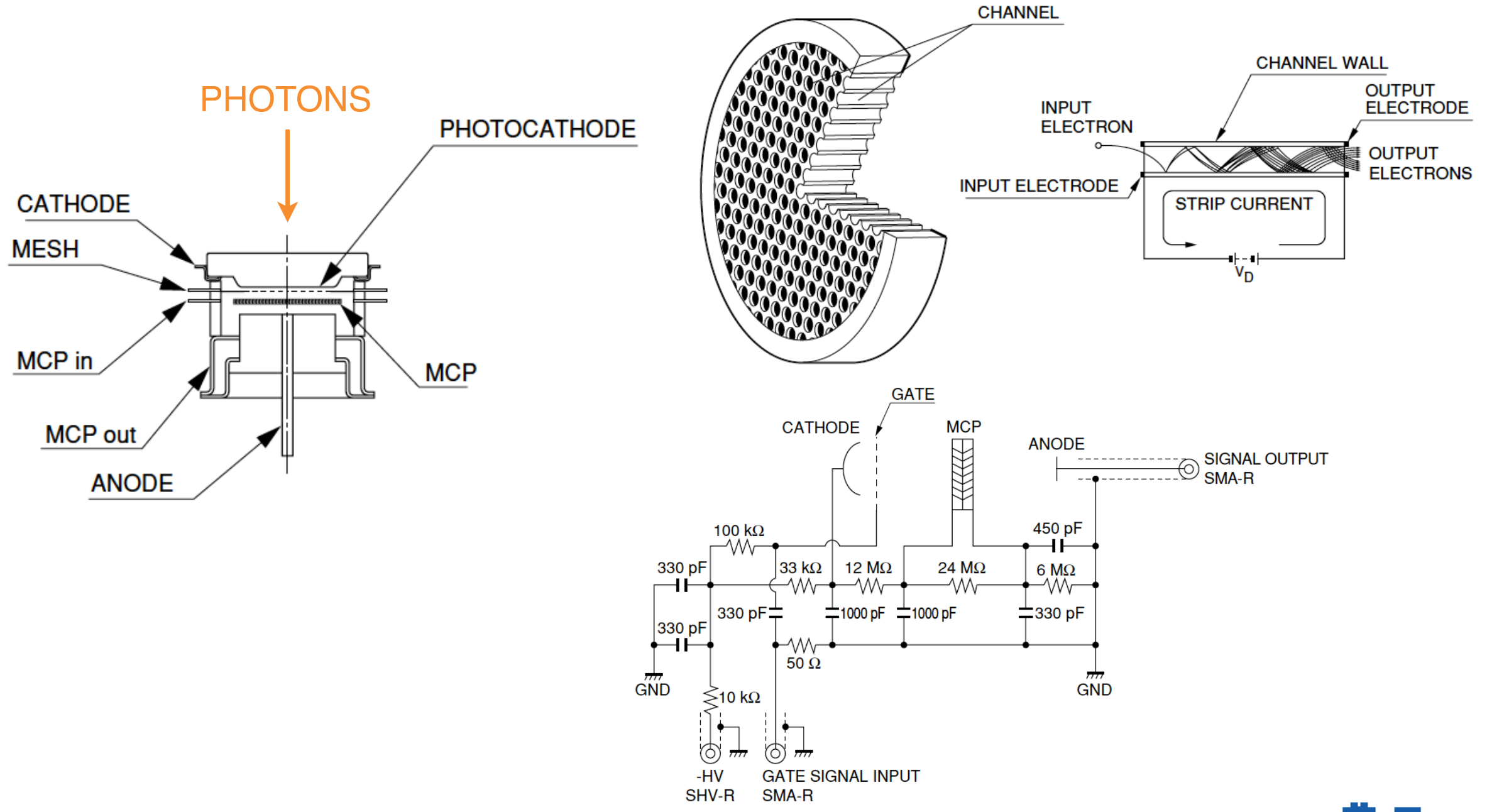


\section{Previous observations of discrete steps in photon flux}
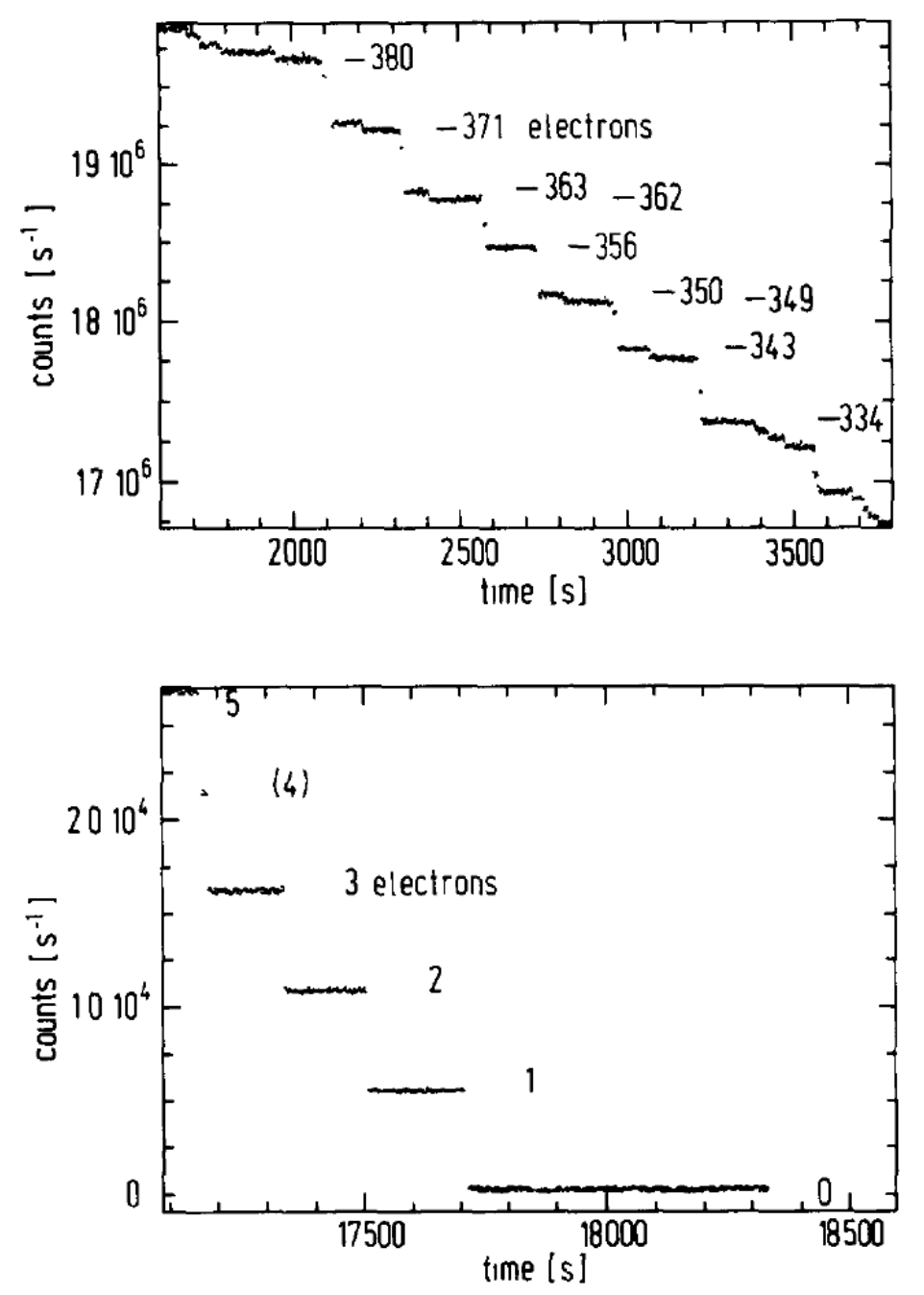

Pinayev et al., NIMA 341, 17 (1994)

Riehle et al., NIMA 268, 262 (1988)

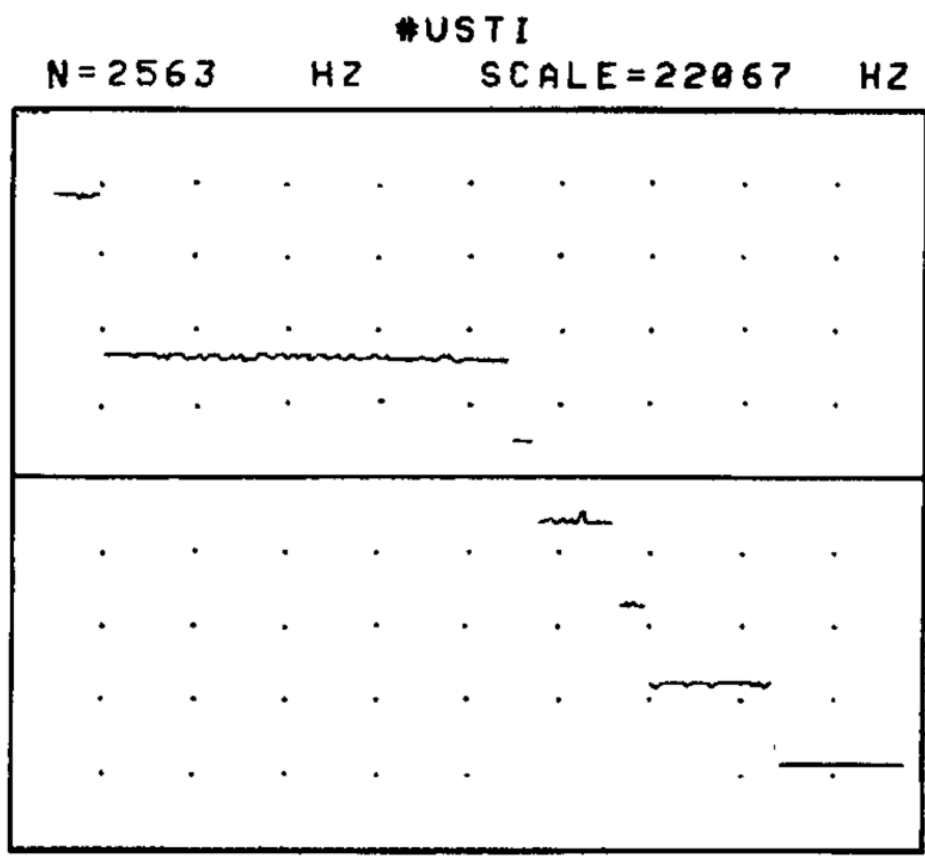

BESSY storage ring 